ORIGINAL

\title{
Suplementación con balanceado comercial en crías vacunas lactantes bajo sistema doble propósito
}

\section{Supplementing double purposed lactating calves with commercial feed}

\author{
Esperanza Prieto M, ${ }^{1 *}$ M.Sc, Donicer Montes ${ }^{1}{ }^{1}$ Esp, Leonardo Lara M, ${ }^{2}$ Zoot, \\ Rosa Ríos $\mathrm{H}^{2}$ Zoot.
}

\begin{abstract}
${ }^{1}$ Universidad de Sucre, Facultad de Ciencias Agropecuarias, Departamento de zootecnia, Sincelejo, Colombia. ${ }^{2}$ Ejercicio particular. *Correspondencia: esperanza.prieto@unisucre.edu.co
\end{abstract}

Recibido: Noviembre 3 de 2009; Aceptado: Julio 26 de 2010.

\section{RESUMEN}

Objetivo. Evaluar el efecto que ejerce la suplementación con alimento balanceado sobre la ganancia diaria de peso (GDP) y el consumo de alimento (CA) en terneros lactantes y su impacto económico en una empresa ganadera manejada bajo un sistema vacuno de doble propósito, en el municipio de Sincé, Sucre-Colombia. Materiales y métodos. Se suplementaron 14 crías ( 6 machos y 8 Hembras) entre 3 y 5 meses de edad, con alimento balanceado comercial al $1.5 \%$ del peso vivo (Tratamiento 1 ) y se compararon con un grupo igual sin suplementar (Tratamiento Testigo), durante 113 días en época de lluvias. La GDP y CA fueron evaluados mediante prueba t de Student. Se determinó Relación beneficio: costo y rentabilidad. Resultados. La GDP de las crías lactantes fue afectada de manera altamente significa por la suplementación con alimento balanceado comercial $(p<0.05)$ siendo en las crías suplementadas $99.77 \%$ ( $870.97 \mathrm{gr}$ ) superior a las crías no suplementadas (435.96 gr.) debido al aporte de nutrientes de alta digestibilidad por parte del suplemento. El sexo de las crías no afectó la ganancia diaria de peso de los terneros dentro de cada tratamiento $(p>0.05)$, encontrándose el mismo resultado para el consumo de alimento balanceado $(p>0.05)$ explicando así la no diferencia estadística en la GDP entre sexos. El análisis económico confirmó que la estrategia alimenticia es viable al presentarse una relación B:C del 1.1. y una rentabilidad trimestral del 9.27\%. Conclusiones. La suplementación de terneros lactantes es una práctica viable, que permite obtener ganancias adicionales.

Palabras clave: Terneros, suplementación, ganancia de peso, alimento, rentabilidad. 


\section{ABSTRACT}

Objective. To evaluate the effect over the daily weight gain (DWG) and feed consumption (FC) caused by supplementing with commercial feed, on lactating calves and its economic impact in a cattle ranching company managed under a double purpose cattle system. Materials and methods. Fourteen calves were supplemented ( 6 males and 8 females) between 3 and 5 months old, with commercial balanced feed at $1.5 \%$ of live weight (Treatment 1 ) and they were compared to an equal in sex group which was not supplemented (control Treatment), for 113 days, during rainy season. The data for DWG and FC were statistically analyzed through a t Student test. The cost-benefit relationship and profitability were determined. Results. The DWG of the lactating calves was significantly affected by the supplementation with commercial feed $(p<0.05)$ being the supplemented calves $99.77 \%(870.97 \mathrm{~g})$ higher to those non-supplemented $(435.96 \mathrm{~g}$.) due to the nutrient contribution and high digestibility of the supplement. The gender of the calves did not affect the daily weight gain $(p>0.05)$, or the consumption of the commercial feed $(p>0.05)$, within each treatment, thus explaining the statistical non-difference in DWG among genders. The economical analysis confirmed that the feeding strategy is viable when there is a relation $B$ : C of 1.1 and a trimester profitability of $9.27 \%$. Conclusions. Supplementing lactating cattle calves is a reasonable practice that allows gaining additional profit.

Key words: Lactating cattle calves, supplementation, weight gain, feeding, profitability.

\section{INTRODUCCION}

La producción bovina en Colombia juega un papel importante para la economía nacional aportando el $66 \%$ del PIB pecuario, con un inventario de 23'000.000 cabezas distribuidas de la siguiente manera: $38 \%$ en el sistema doble propósito, 60\% para cría, levante y ceba y el $2 \%$ para lecherías especializadas, siendo estos los principales sistemas de producción manejados en el país (1).

En las ganaderías manejadas bajo el sistema doble propósito, los terneros lactantes representan uno de los grupos o categorías más importantes, debido a que de allí surgirán las futuras hembras de reemplazo y los machos destetos que representan uno de los componentes de ingreso económico. Uno de los principales problemas que se presenta en ésta categoría animal es el desequilibrio en el aporte de nutrientes necesarios para un adecuado crecimiento; la principal causa es el mal manejo alimenticio que se les ofrece a los terneros, debido a varios factores como son: por una parte al exhaustivo ordeño al que son sometidas las madres, las cuales por tener un alto componente Bos taurus dejan menos leche residual al ternero. Por otro lado las crías menores de 4 meses, solo toman la leche residual después del ordeño y permanecen con sus madres entre 5 y 7 horas diarias, posteriormente son encerradas en horas de la tarde (1:00 p.m.) en los corrales, sin ninguna oferta de alimento, hasta el ordeño del día siguiente (6:00 a.m.) y/o son sometidas a un pastoreo en los potreros mas cercanos a la casa, presentando estos en la mayoría de los casos, forraje en muy baja cantidad y calidad.

Lo anterior ocasiona problemas de subnutricion durante esta etapa productiva, conllevando a bajas ganancias diarias de peso y bajos pesos al destete (inferiores a $130 \mathrm{Kg}$ ), mayor incidencia de problemas sanitarios, alta mortalidad y un precio reducido al momento de la venta. También es importante destacar que de este grupo de animales surgen, normalmente, las futuras hembras de reemplazo, que representan, en gran medida, el futuro económico de la empresa, en este caso los futuros vientres llegan a una edad tardía a la pubertad y 
por consiguiente con alta edad al primer parto, dando pocos terneros durante su vida productiva.

El objetivo de esta investigación fue evaluar el efecto que ejerce la suplementación con alimento balanceado comercial sobre la ganancia diaria de peso y su viabilidad económica, en terneros lactantes manejados bajo un sistema vacuno de doble propósito en condiciones de sabana en el departamento de Sucre.

\section{MATERIALES Y MÉTODOS}

Tipo de estudio. Se realizó un estudio de tipo experimental con asignación aleatorizada para evaluar dos tratamientos en un lote de hembras y uno de machos, con ocho repeticiones para las hembras y seis para los machos en cada tratamiento, para un total de 28 unidades experimentales, observadas durante 113 días.

Localización. El trabajo se llevó a cabo en la empresa ganadera El Nido, municipio de Sincé, subregión sabanas del departamento de Sucre. Su posición geográfica está determinada entre los $9^{\circ} 14^{\prime} 45^{\prime \prime}$ de latitud Norte y $75^{\circ} 08^{\prime} 58^{\prime \prime}$ de longitud Oeste, en el kilómetro 35 de la entrada a Sincé desde San Juan de Betulia. Los datos meteorológicos de la zona afirman que el lugar se encuentra de acuerdo con la clasificación de Montes (2) en bosque seco tropical (bs-T) a 125 m.s.n.m., con temperatura promedio de $28^{\circ} \mathrm{C}$, humedad relativa de $75 \%$ y precipitación media anual de 1100 m.m., distribuidos entre los meses de marzo y noviembre.

La empresa ganadera posee 36 hectáreas distribuidas en 12 potreros, de los cuales tres se encuentran ocupados con pastos de corte como elefante morado, elefante enano y caña dulce, los 9 restantes están en praderas. La principal fuente de alimentación consiste en praderas conformadas por una mezcla de gramíneas y leguminosas siendo el pasto colosuana (Bothriochloa pertusa) el predominante. Se cuenta con agua subterránea para el sistema de riego, agua de jagüeyes y agua de acueducto para el consumo de los animales.
Población y muestra. Como unidades experimentales se utilizaron un total de 28 terneros lactantes, integrados por 12 machos y 16 hembras, todos cruzados resultantes del sistema doble propósito con componentes raciales desde 3/4 Bos taurus hasta 3/4 Bos indicus, con participación de las razas Holstein, Pardo Suizo, Gyr, Brahman y Guzerat. Los terneros fueron clasificados y seleccionados lo mas homogéneos posible con una edad entre 3 y 5 meses y un peso promedio de $79.05 \mathrm{Kg}$ para los machos y $75.55 \mathrm{Kg}$ para las hembras.

Se evaluaron los siguientes tratamientos sobre el desempeño productivo:

Tratamiento Control $\left(\mathbf{T}_{0}\right)$ : Crías no suplementadas.

Tratamiento $\mathbf{1}\left(\mathbf{T}_{\mathbf{1}}\right)$ : Crías suplementadas con alimento balanceado comercial $(1.5 \%$ del Peso Vivo).

Las unidades experimentales fueron asignadas al azar en los diferentes tratamientos, de tal forma que cada uno quedo representado por 14 crías, conformado por 6 machos y 8 hembras.

La evaluación se llevó a cabo durante un periodo experimental de 113 días en la época de lluvia, de los cuales 14 correspondieron a la fase de acostumbramiento y 99 a la evaluación y toma de datos.

Se verificó que todos los terneros mamaran la leche residual luego del ordeño de cada vaca durante media hora, finalizada esta actividad, las crías salían en compañía de sus madres al potrero asignado para vacas paridas donde encontraba agua y mezcla mineral comercial al $8 \%$ de P.

Los terneros evaluados fueron separados todos los días de sus madres a las 11:00 a.m., y llevados a pastoreo. Para esto se utilizaron 2 potreros de 0.9 ha $(c / u)$ para cada tratamiento, cada uno de estos potreros se subdividió en 2, manejando un sistema de pastoreo alterno, con 14 días de ocupación y 14 días de descanso, permaneciendo las crías en su respectivo potrero el resto del día. Las crías del tratamiento 1 fueron trasladadas a las 4:00 p.m., a los corrales donde se les brindó 
la suplementación, ofreciendo $1.5 \%$ del peso vivo, luego de saciarse eran dirigidas nuevamente a los potreros donde pasaban el resto de la noche hasta el ordeño del día siguiente. Una vez retirados los terneros se pesó el alimento residual. La cantidad de alimento se ajustó cada quince días y se ofreció de manera individual en comederos de madera con medidas de $60 \mathrm{~cm}$ de ancho $x 70 \mathrm{~cm}$ de largo y $30 \mathrm{~cm}$ de fondo.

El suplemento ofrecido fue en forma de pellet para iniciación de terneros en zonas tropicales, con suministro a partir de los 10 días de edad hasta los 9 meses. La composición química del alimento se presenta en el tabla 1.

Tabla 1. Composición nutricional del suplemento alimenticio suministrado a las crías evaluadas en el tratamiento $1\left(T_{1}\right)$.

\begin{tabular}{lc}
\hline \multicolumn{2}{c}{ Análisis garantizado } \\
\hline Ceniza máxima (\%) & 10.00 \\
Fibra máxima (\%) & 10.00 \\
Gasa mínima (\%) & 3.00 \\
Humedad máxima (\%) & 12.00 \\
Proteína mínima (\%) & 18.00 \\
\hline
\end{tabular}

Recolección procesamiento y análisis de la información. Se evaluaron las siguientes variables: Ganancia diaria de peso, consumo diario de suplemento, relación beneficiocosto, utilidad neta y rentabilidad.

Ganancia diaria de peso. Los terneros fueron pesados cada quince días, de manera individual, antes de que cada ternero tuviera contacto con la madre durante el ordeño, este pesaje se hizo utilizando una báscula ganadera.

Consumo diario de alimento. Diariamente se determinó el consumo del suplemento o alimento balanceado por parte de las crías del tratamiento 1 , que resultó de la diferencia entre la cantidad ofrecida y los sobrantes o rechazo.

Debido a que los dos tratamientos experimentales se sometieron al pastoreo se determinó la disponibilidad de materia seca y composición botánica a la entrada y salida de cada pastoreo.
Adicionalmente, se evaluó la composición química del forraje disponible y la muestra se colectó a través del método de simulación de pastoreo ("hand plucking"). Se determinó porcentajes de materia seca (MS), proteína bruta (PB), cenizas (C) y materia orgánica (MO) por el método AOAC (1984), porcentajes de fibra detergente neutro (FDN), fibra detergente ácido (FDA) y lignina $($ LDA $)(3)$.

Indicadores económicos. Para el cálculo de los indicadores económicos, se tuvo en cuenta los costos e ingresos adicionales por efectos de la suplementación, es decir los egresos por consumo del alimento balanceado comercial y los ingresos por la ganancia total de peso adicional que obtuvieron las crías suplementadas frente a las no suplementadas.

Análisis de los resultados. La ganancia diaria de peso y el consumo de alimento fueron analizadas a través de la prueba t Student, para dos colas a un nivel de confianza del 95\% (4), considerando los efectos simples de los tratamientos en donde se compararon las medias obtenidas para cada variable por tratamiento y sexo. Para la evaluación económica, se determinó la relación beneficio costo, la utilidad neta y la rentabilidad. El precio por $\mathrm{kg}$ de peso fue considerado de acuerdo al precio de venta de animales anteriores en la finca.

\section{RESULTADOS}

Ganancia diaria de peso (GDP). La GDP de las crías lactantes fue afectada de manera altamente significa por la suplementación con alimento balanceado comercial ( $p<0.05)$, siendo de $870.97 \mathrm{~g} /$ día para las crías suplementadas y de $435.96 \mathrm{~g} /$ día para las crías no suplementadas.

El sexo de las crías no afectó la ganancia diaria de peso de los terneros dentro de cada tratamiento $(p>0.05)$, sin embargo tendió a ser mayor en los machos.

El análisis estadístico de las crías del mismo sexo pero de diferentes tratamientos mostraron diferencias altamente 
significativas $(p<0.05)$ lo que corrobora el efecto positivo de la suplementación en la ganancia diaria de peso, de esta manera tanto machos como hembras del tratamiento 1 , fueron superiores a las crías del mismo sexo del tratamiento control (Tabla 2 ).

Tabla 2. GDP por sexo de las crías suplementadas $\left(T_{1}\right)$ y No suplementadas $\left(T_{0}\right)$ bajo el sistema de doble propósito.

\begin{tabular}{lcc}
\hline \multicolumn{1}{c}{ SEXO } & $\mathbf{T}_{1}$ & $\mathbf{T}_{\mathbf{0}}$ \\
\hline Macho & $930.42(\mathrm{a})$ & $454.93(\mathrm{~b})$ \\
Hembras & $846.38(\mathrm{a})$ & $421.74(\mathrm{~b})$ \\
\hline
\end{tabular}

Consumo de alimento. Para el consumo de alimento balanceado por parte de los terneros del tratamiento 1 , no existió diferencia significativa entre machos y hembras $(p>0.05)$, de otra parte, el consumo de suplemento se encontró entre 1.35 y $1.29 \%$ del peso vivo, sin conseguir el $1.5 \%$ (Tabla 3 ).

Tabla 3. Consumo diario de balanceado comercial (g.) por parte de las crías lactantes suplementadas $\left(T_{1}\right)$ bajo el sistema de doble propósito.

\begin{tabular}{lccc}
\hline & Macho & Hembra & Media \\
\hline $\begin{array}{l}\text { Consumo de } \\
\text { Balanceado (g) }\end{array}$ & 1508.60 a & 1495.29 a & 1501.94 \\
$\begin{array}{l}\text { Consumo de } \\
\text { Balanceado (\%PV) }\end{array}$ & $1.35 a$ & 1.29 a & 1.32 \\
\hline
\end{tabular}

a, letras iguales entre filas, no presentan diferencia significativa $(p>0.05)$

En promedio los teneros consumieron $1500 \mathrm{~g}$ de alimento balanceado diarios de donde obtuvieron un consumo de $1.31 \mathrm{~kg}$ de MS y $267.79 \mathrm{~g}$ de proteína cruda de alta digestibilidad lo que significa que el suplemento aportó el $50 \%$ de la materia seca, el $44.12 \%$ de la proteína cruda y el $41.8 \%$ de energía digestible (3.75 Mcal), dentro de los requerimientos nutricionales de las crías en esta etapa.

Al estimar el consumo de leche residual (como el $30 \%$ de la producción de la vaca) y calcular el consumo de forraje, se pudo observar que el consumo de leche residual entre los terneros suplementados y no suplementados fue similar, pues este parámetro depende de la capacidad productora de las madres el cual presentó un comportamiento parecido; no obstante, el consumo de alimento balanceado por parte de los terneros del T1, disminuyó el consumo de forraje durante el pastoreo en este grupo de animales (Tabla 4).

Tabla 4. Consumo diario de leche y forraje durante el pastoreo de las crías vacunas lactantes no suplementadas $\left(\mathbf{T}_{\mathbf{0}}\right)$ y suplementadas $\left(\mathbf{T}_{1}\right)$ bajo el sistema DP.

\begin{tabular}{lcc}
\hline & $\mathbf{T}_{0}$ & $\mathbf{T}_{1}$ \\
\hline Consumo de leche (Lt.) & 1.08 & 1.1 \\
Consumo de forraje (\% PV) & 7.0 & 4.3 \\
\hline
\end{tabular}

Al considerar el consumo de nutrientes en el alimento balanceado, la leche y el forraje (Tabla 5) se puede afirmar que los terneros suplementados presentaron una dieta bastante completa que se aproximó a los requerimientos nutricionales de las crías , lo que explica en gran parte los buenos resultados encontrados en cuanto a GDP se trata.

Tabla 5. Consumo estimado de nutrientes por parte de las crías vacunas lactantes no suplementadas $\left(T_{0}\right)$ y suplementadas $\left(T_{1}\right)$.

\begin{tabular}{lcccccc}
\hline & \multicolumn{2}{c}{ M.S (Kg.) } & \multicolumn{2}{c}{ PC (g.) } & \multicolumn{2}{c}{ E.D (Mcal) } \\
\hline & $\mathrm{T}_{0}$ & $\mathrm{~T}_{1}$ & $\mathrm{~T}_{0}$ & $\mathrm{~T}_{1}$ & $\mathrm{~T}_{0}$ & $\mathrm{~T}_{1}$ \\
Balanceado & 0 & 1.31 & 0 & 267.79 & 0 & 3.75 \\
Leche & 0.162 & 0.165 & 5.67 & 5.77 & 0.25 & 0.26 \\
Forraje & 2.14 & 1.6 & 182.2 & 144 & 3.5 & 2.71 \\
\hline Total & $\mathbf{2 . 3}$ & $\mathbf{3 . 0}$ & $\mathbf{1 8 7 . 8 7}$ & $\mathbf{4 1 7 . 5 4}$ & $\mathbf{3 . 7 5}$ & $\mathbf{6 . 7 2}$ \\
\hline Requerimientos NRC $\mathbf{1 0 0 0} \mathbf{g}$ & & 2.8 & & 460 & & 7.65 \\
Requerimientos NRC $\mathbf{8 0 0} \mathbf{g}$ & & 2.7 & & 434.5 & & 7.25 \\
\hline
\end{tabular}


Viabilidad económica de la suplementación sobre la ganancia diaria de peso (GDP) en crías lactantes. En la tabla 6, se muestra el consumo total de suplemento balanceado comercial de las crías del tratamiento 1 , así como su equivalencia en dinero, lo que representa la inversión adicional a los costos de producción realizados en la empresa ganadera en comparación con el tratamiento control, que para efectos de la investigación reflejó el comportamiento tradicional de la finca. En este sentido el gerente ganadero invirtió \$ $1.882 .085,6$ en $2.352,61 \mathrm{~kg}$ de alimento balanceado el cual consumieron las 14 crías en un periodo de 113 días evaluados.

Tabla 6. Costo de la suplementación con balanceado comercial en crías vacunas lactantes durante el periodo experimental.

\begin{tabular}{lccc}
\hline & Machos & Hembras & Total \\
\hline $\begin{array}{l}\text { Total consumo } \\
\text { (Kg.) }\end{array}$ & $1.069,62$ & $1.282,99$ & $2.352,61$ \\
$\begin{array}{l}\text { Costo Alimento } \\
(\$ / \mathrm{Kg})\end{array}$ & $\$ 800,00$ & $\$ 800,00$ & $\$ 800,00$ \\
\multicolumn{2}{c}{ Total Costo Alimento } & $\mathbf{\$ 1 . 8 8 2 . 0 8 5 , 6 0}$ \\
\hline
\end{tabular}

Los ingresos adicionales por efectos de la suplementación aparecen en la tabla 7 donde se observa que las crías suplementadas obtuvieron $685.5 \mathrm{~kg}$ de peso adicionales a las crías no suplementadas (1369.5 $684 \mathrm{~kg}$ ), esto representado en un ingreso adicional de $\$ 2.056 .500$ tomando como precio $\$ 3000$ por $\mathrm{kg}$ de peso vivo.
La viabilidad económica adicional por efecto de la suplementación con alimento balanceado, se puede apreciar en el tabla 8.

Tabla 8. Cálculo de la viabilidad económica adicional por efecto de la suplementación con balanceado comercial en Crías lactantes.

\begin{tabular}{lc}
\hline $\begin{array}{l}\text { Rentabilidad neta adicional por } \\
\text { suplementación }(\%)\end{array}$ & 9.27 \\
$\begin{array}{l}\text { Relación Beneficio: Costo por gasto } \\
\text { adicional }\end{array}$ & 1.1 \\
$\begin{array}{l}\text { Utilidad neta por efecto de } \\
\text { suplementación }(\$)\end{array}$ & 174.414 .40 \\
$\begin{array}{l}\text { Utilidad por } \mathrm{Kg} . \mathrm{de} \text { alimento } \\
\text { Consumido }(\$ / \mathrm{Kg} .)\end{array}$ & 74.14 \\
\hline
\end{tabular}

\section{DISCUSIÓN}

Ganancia diaria de peso (GDP). Las crías suplementadas con alimento balanceado comercial superaron en $99.77 \%$ a las crías no suplementadas en ganancia de peso. De acuerdo con lo expuesto por Combellas (5), los alimentos balanceados tienen un efecto mejorador sobre las variables productivas por la disponibilidad de nutrientes que estos poseen y por la calidad energética que son capaces de proveer a los rumiantes, que un pasto en clima tropical difícilmente suministraría, siendo el limitante en un sistema de pastoreo en el trópico.

A pesar del resultado anteriormente expuesto, las crías suplementadas no alcanzaron ganancias diarias de peso de $1000 \mathrm{~g}$ diarios, esto pudo deberse a las actividades de descorne y marcaje a que fueron sometidos todos los animales, dentro del $3^{\circ}$ al $5^{\circ}$ pesaje, en donde las

Tabla 7. Ingreso adicional en crías lactantes Suplementadas con balanceado comercial durante el periodo experimental.

\begin{tabular}{|c|c|c|c|c|c|c|c|}
\hline & KG & Machos & Hembras & $\begin{array}{l}\text { Total } \\
(\mathrm{Kg})\end{array}$ & $\begin{array}{c}\text { Ganancia } \\
\text { Total (Kg) }\end{array}$ & $\begin{array}{c}\text { Ganancia } \\
\text { Adicional } \\
(\mathrm{Kg})\end{array}$ & $\begin{array}{c}\text { Ingreso } \\
\text { Adicional } \\
(\$)\end{array}$ \\
\hline \multirow[t]{2}{*}{ Suplementados } & Inicial & 467 & 596 & 1063 & 1369,5 & \multirow{4}{*}{685.5} & \multirow{4}{*}{2.056 .500} \\
\hline & Final & 1093 & 1339.5 & 2432.5 & & & \\
\hline \multirow{2}{*}{$\begin{array}{c}\text { No } \\
\text { Suplementados }\end{array}$} & Inicial & 482 & 613 & 1095 & \multirow{2}{*}{684} & & \\
\hline & Final & 788 & 991 & 1779 & & & \\
\hline
\end{tabular}


ganancias de peso cayeron entre un 33.8 y $45.2 \%$. Este descenso pudo ser causado por efectos de la acción de corticoides en el estrés y por el dolor que experimentaron los animales a través de la cura de heridas que se realizaron en este periodo. Se pudo notar, un incremento de la GDP para los animales en los dos tratamientos al sanar completamente las heridas de las crías.

Varios investigadores han evaluado el efecto de la suplementación sobre la ganancia diaria de peso en crías lactantes siendo los resultados en la mayoría de los casos satisfactorios a favor de la suplementación. Martínez et al (6), estudiaron el efecto de tres niveles de suplementación $(0.5,1.0 \mathrm{y}$ $1.5 \%$ del peso vivo) con alimento balanceado vs un tratamiento control, sobre la GDP en crías lactantes mestizas manejadas bajo un sistema de doble propósito. Los resultados encontrados mostraron efectos similares a los de la presente investigación, siendo la alimentación con balanceado en los tres niveles evaluados, las que presentaron los valores mas altos en ganancia diaria de peso, con diferencias altamente significativas $(p<0.01)$ sobre los terneros no suplementados. La GDP también fue afectada por los diferentes niveles de suplementación, presentando los mejores resultados los niveles al $1 \mathrm{y}$ al $1.5 \%$, sin diferencia significativa $(p>0.05)$ entre estos, pero ambos superiores a los otros con diferencia significativa, siendo la opción más atractiva económicamente, la suplementación al $1 \%$, con una rentabilidad de $21 \%$ en un periodo de 8 meses hasta el destete de los animales.

Reza et al (7), Blanco (8), estudiaron el efecto de la suplementación del ternero lactante bajo un sistemas vacuno de doble utilidad; en todos los casos, los terneros suplementados obtuvieron mejores resultados productivos en GDP cuando los compararon con los terneros no suplementados.

Resultados diferentes fueron encontrados por Cero et al (9), quienes estudiaron el efecto de la suplementación en terneros Cebú cubano, no encontrando diferencia significativa entre los terneros suplementados y no suplementados, atribuyendo dicho efecto a la capacidad genética limitada de las crías suplementadas, la cual no les permitió mantener e incrementar la producción frente a los no suplementados y por ende la suplementaciòn no fue viable económicamente.

La no diferencia en la GDP por sexo, puede atribuirse a que las crías vacunas hembras y machos en etapa media de lactancia, dependiendo de las condiciones de manejo, presentan un crecimiento homogéneo, efecto que podría estar explicado por la interacción genotipo ambiente (10). A pesar de lo anterior muchos investigadores han encontrado efectos diferentes a los aquí reportados. Por ejemplo, Osorio (11), obtuvo $35 \mathrm{~kg}$ más al destete y $105 \mathrm{~g}$ más en GDP en crías machos con relación a las hembras siendo el promedio de machos de $205,9 \mathrm{~kg}$ al destete y $701.2 \mathrm{~g}$ en GDP con relación a las hembras con $180 \mathrm{~kg}$ al destete y $551 \mathrm{~g}$ en GDP. Por otra parte, Álvarez (12), señala una ventaja de 6 a $9 \%$ a favor de los machos para la GDP en la lactancia frente a las hembras, manejadas bajo un sistema de doble propósito y pastoreo rotacional.

\section{Consumo de alimento balanceado.} La no diferencia en el consumo promedio de alimento balanceado entre machos y hembras, podrían en parte explicar la no diferencia estadística en la GDP entre sexos dentro de este tratamiento.

El consumo de alimento balanceado y el aporte de nutrientes que este hizo, explica la eficiencia productiva de los terneros suplementados frente a los no suplementados; por un lado, se satisface en parte los requerimientos nutricionales para esta etapa y por otro lado dentro de su comportamiento ingestivo, disminuiría el tiempo dedicado a la búsqueda de alimento o pasto e incrementaría el tiempo de reposo y por ende su eficiencia metabólica, esto reforzado por una alta digestibilidad del alimento consumido.

El consumo de alimento balanceado no alcanzó a llegar al $1.5 \%$ del peso vivo en los diferentes sexos; siendo de $1.32 \%$ en promedio; lo anterior debido posiblemente 
a la época de invierno donde hay mayor disponibilidad de forraje, mayor producción de leche y mayor consumo de leche por parte del ternero. Es posible que en verano, donde disminuye la producción de forraje, se logre un consumo del $1.5 \%$ del peso vivo.

El consumo de alimento balanceado de los terneros del T1, disminuyó el consumo de forraje durante el pastoreo. Varios investigadores coinciden con lo anterior (13-15), quienes encontraron que la suplementación energética $\mathrm{y} / \mathrm{o}$ proteica disminuyen el consumo de materia seca en el forraje, así como tiende a reducir el tiempo dedicado a la actividad de pastoreo, lo cual incrementa la tasa metabólica de los animales y por ende su eficiencia productiva. En todos los casos anteriores, a medida que aumentó el nivel de inclusión del suplemento con relación al peso vivo disminuyó el consumo de forraje. Por lo anterior es posible que adicionalmente la suplementación permita aumentar la carga animal en estos potreros.

Viabilidad económica de la suplementación sobre la ganancia diaria de peso (GDP) en crías lactantes. Las grandes ventajas biológicas (GDP) que ofreció el alimento balanceado comercial a las crías del tratamiento uno frente a las del tratamiento control, mostró un efecto proporcional en los indicadores económicos para la viabilidad de la estrategia evaluada (Tabla 8), en tal sentido que la rentabilidad de la inversión adicional por efectos de la suplementación alcanzó un 9.27\% durante el periodo evaluado (113 días), encontrándose por encima a la tasa de interés bancaria nacional ${ }^{1}$. El resultado indicó que por cada \$100 invertidos se obtuvo \$9.27 de utilidad adicional, esto confirma que es viable económicamente la implementación de esta estrategia alimenticia; por otro lado, la relación beneficio:costo, solo llegó a 1.1 que a pesar de no arrojar perdidas si está algo estrecha, lo que se refleja en una utilidad bruta adicional baja siendo de \$ 174.414 en 113 días evaluados. Este efecto puede mitigarse realizando una reducción en el nivel de suplementación llegando al $0.5 \%$ (16) o al $1.0 \%$ (6) del peso, pues según estos investigadores, estos niveles de suplementación fueron los favorables económicamente.

Bravo y Bracho 2000 (17), encontraron que la suplementación al $0.5 \%$ representó una disminución del $84.52 \%$ con respecto al $1.5 \%$, asimismo, la suplementación permitió obtener una rentabilidad del 25\% anual, considerándose bastante buena y estando por encima a la encontrada en la presente investigación.

En conclusión, la suplementación con alimento balanceado comercial a crías lactantes manejadas bajo el sistema de doble propósito y en las condiciones tropicales de sabanas evaluadas, permitió duplicar la ganancia diaria de peso frente a las crías no suplementadas. El sexo de las crías evaluadas no afectó la ganancia diaria de peso durante el periodo experimental dentro de cada tratamiento. El consumo de alimento balanceado comercial fue estadísticamente el mismo para las crías machos y hembras del tratamiento suplementado.

El análisis económico realizado a la suplementación de terneros lactantes manejados bajo el sistema de doble propósito, confirmó que es una practica viable, que permite obtener una ganancia de dinero extra, en el ciclo de producción, al presentarse una rentabilidad por encima a la tasa de interés bancaria y margen positivo de utilidad adicional. Sin embargo el máximo beneficio se consigue a largo plazo, ya que las hembras, podrían llegar mas temprano a una vida reproductiva, al presentar un crecimiento mucho mas rápido que las no suplementadas; del mismo modo los machos llegarían más jóvenes al faenamiento incrementando la calidad de la canal.

Se recomienda la evaluación de diferentes niveles de suplementación con alimento balanceado comercial a crías lactantes con el fin de encontrar las más viable, no solamente productiva sino económicamente aplicable. Continuar con el monitoreo de los pesajes de los animales y la suplementación y de esta forma conocer su impacto en la llegada a la pubertad y edad al primer parto en las hembras. 


\section{REFERENCIAS}

1. FEDEGAN. Plan estratégico de la ganadería 2019, Bogotá: FEDEGAN; 2006: 23-64.

2. Montes S. Bosque seco tropical. Colombia: Editorial banco de occidente; 2006.

3. Slavin J. Impact of the Proposed Definition of Dietary Fiber on Nutrient Databases. J Food Compost Anal 2003; 16(3):287-291.

4. SPSS, Version 14 for Windows. [programa informático en CD-ROM]. Chicago: SPSS Inc.; 2006.

5. Combellas L J. Alimentación de la Vaca de Doble Propósito y sus crías. Maracaybo, Venezuela: Fundación INLACA; 1998.

6. Martínez M, Bravo J, Betancourt M, Morán V. Efecto de la suplementación sobre el crecimiento de becerros mestizos en la época seca. Zootecnia Trop, 2001; 19(1):31-42.

7. Reza GS, Cuadrado $\mathrm{CH}$, Martínez AJ, Alvarado AL, Abuabara PY, Torregroza SL. Análisis técnico-económico de un manejo de alimentación para bovinos lactantes en el sistema doble propósito en el Valle del Sinú. Carta Fedegán 2003; (78):65-69.

8. Blanco M. Repercusión del destete precoz y la suplementación sobre las pautas de crecimiento y desarrollo de los terneros. [Tesis doctoral]. España: Universidad de Lleida, Departamento de producción; 2007. [en línea] URL Disponible en http://www.tdx.cesca.es/ TESIS_UdL/AVAILABLE/TDX-0216108180813/Tmba1de1.pdf.

9. Cero AR, Guevara VG, Corvison RM, Del toro $A$. Factores genéticos y ambientales que afectan el peso y la ganancia media al destete en el cebú cubano bermejo. [Trabajo de grado]. Cuba: Universidad de Camagüey, Facultad de Ciencias Agropecuarias; 1999.

10. Sandoval $E$, Valle $A$, Jiménez $D$, Oswaldo Márquez O. Evaluación de pesos al nacer y crecimiento en becerros doble propósito amamantados con vacas nodrizas durante la etapa de lactantes. Zootecnia Trop 2005; 23(1):1-16. [en línea]. URL disponible en: http:// www.sian.inia.gob.ve/repositorio/ revistas_ci/ZootecniaTropical/zt2301/ arti/sandoval_e.htm

11. Osorio M, Segura J. Factores ambientales que afectan la curva de crecimiento de becerros de un sistema de doble propósito en el trópico. Villahermosa, Tabasco, Mexico: Memoria XIV Reunión Científico-Tecnológica, Forestal y Agropecuaria. INIFAP-Produce; 2001.

12. Álvarez P. Estimación de parámetros productivos en hatos de ganado bovino. [Tesis de Maestría]. Caracas, Venezuela: Universidad Central de Venezuela, Facultad de Ciencias Veterinarias; 2001.

13. Patiño RM, Fischer $V$, Balbinotti $M$, Moreno C, Ferreira EX, Vinhas R, et al. Efeitos do fornecimento níveis crescentes de suplemento energético sobre o comportamento ingestivo diurno de becerros de corte em pastejo. [Tesis Magíster]. Brasil: Universidad Federal de Pelotas, Facultad de zootecnia; 2001.

14. Aguilar N, Slanac A, Balbuena O. Comportamiento ingestivo en vaquillas cruza Cebú en pastoreo, que reciben suplementación energético proteica. Argentina: Estación experimental INTA; 2002.

15. Velásquez J. Conducta de pastoreo en ganado bovino. El Cebú 2003; 333:1-16. 
16. Martínez M, Bravo J, Betancur M, Moran V. Efecto de la suplementación sobre el crecimiento de becerros mestizos en la época seca. Zootecnia Trop 2001; $19(1): 312-42$.
17. Bravo J, Bracho I. Respuesta de becerros criollo limonero sometidos a diferentes niveles de suplementación: Rev Fac Agron (LUZ) 2000;(13):282-287. 